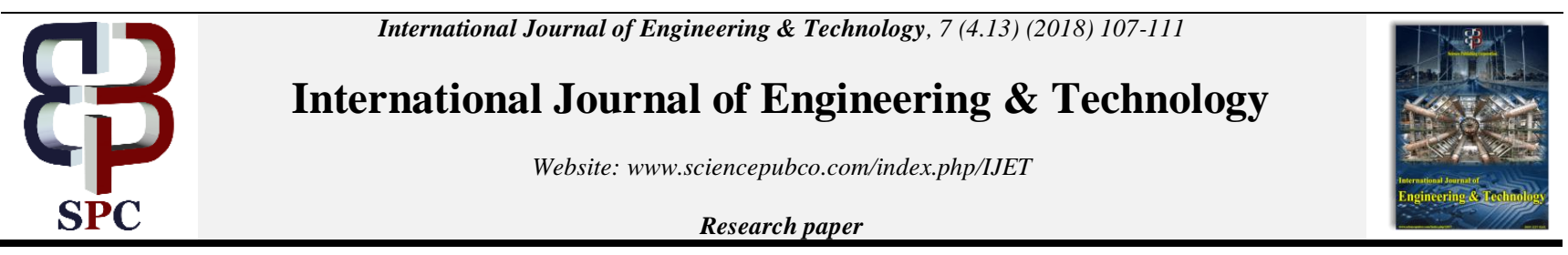

\title{
Investigation of closed compartment moulding for pull-winding process
}

\author{
Abdul Malek Ya'acob ${ }^{1 *}$, Mohamad Daniel Aizat Mohd Razali ${ }^{1}$, Umar Abdillah Anwar ${ }^{1}$, Mohd Arif Hakimi \\ Mohd Radhi ${ }^{1}$, Muhammad Aizat Afifi Ishak ${ }^{1}$, Mulia Minhat ${ }^{1}$, Khairul Dahri Mohd Aris ${ }^{1}$, \\ Muhd Khudri Johari ${ }^{1}$, Casey Teh $^{2}$ \\ ${ }^{1}$ Universiti Kuala Lumpur - Malaysian Institute of Aviation Technology, Dengkil, Selangor, MALAYSIA \\ ${ }^{2}$ TenAsia Corporation Sdn. Bhd., Nilai, Negeri Sembilan, Malaysia \\ *Corresponding author E-mail: abdulmalek@unikl.edu.my
}

\begin{abstract}
This project investigates the closed compartment moulding design for pull-winding process. This is a continuation of an initial study that involved discovering how the permeability effect inside a close compartment in pull-winding processing. The mould has been designed to ensure resin penetration to the preform. The mould design has been divided into three parts for easy installation, maintenance and also cleaning after use. The mould has been tested on an actual production line at TenAsia Sdn. Bhd. The test is conducted by pulling mechanism where the machine pulls the preform winding forward when it is switched on. In this process, the resin is flowing through the hose and it has been sprayed out by the nozzle inside the mould to the preform. The test has been done using 2,4 and 6 nozzles with various pressures to determine which one is the most suitable for penetration and covering up the preform. Based on the results, the most economical and productive design has been identified as spray type with 2 upper and lower nozzles running at an appropriate pressure of 20 psi. This pressure has been chosen based on the area covered on the preform surfaces and time taken to penetrate the preform layer. In conclusion, 20 psi pressure works better due to the resin's deeper penetration in shorter time, less mist and very minimal excess resin.
\end{abstract}

Keywords: closed-compartment moulding; pull-winding; permeability effect; processing line.

\section{Introduction}

In present decades, a big attention has been dedicated to the use of fibre-reinforced composites in various engineering fields [1]. This also includes many aerospace applications. The desire to use these materials is mainly due to their improved stiffness-to-weight and strength-to-weight ratios. With their high demands and popularity in countless requests [2], it has been estimated that more than four million tonnes of fibre-reinforced composites have been produced in 2010 [3]. Among these, about 300,000 tonnes have been fabricated via the 'pull-winding' method [4]. At the finishing stage, the pull-winding is one of the optional processing methods that can be used, which also include resin bath-based impregnation, wet- and dry-tape pull winding, electrostatic deposition, prepreg pull winding and laser-assisted impregnation. For the pull-winding process, the causes to its industry problems can be related to the schematic diagram of the process in Figure 1, where the denotations used are described in Table 1.

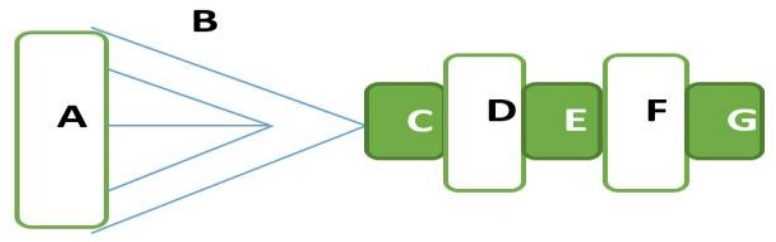

Fig. 1: Schematic drawing in the actual pull-winding processing line
Table 1: Indicators for A-F before the final product $(\mathrm{G})$

\begin{tabular}{c|c|c}
\hline A: Fibre cell & B: Fibre & C: Dry fibre \\
D: Mould & E: Wet fibre & F: Heated die \\
\hline
\end{tabular}

The resin bath-based impregnation method is a predominant method utilized in industry and usually involves the use of thermosetting resin. In this discovery, the "resin system" is utilized to delineate an intimately mixed resin and hardener. Most manufacturers are using traditional way in their pull-winding manufacturing plant, which is the open resin bath. Resin baths of five-liter capacity are often used to achieve the fiber impregnation. The resin is normally manually mixed and deposited into the bath. In general, thermosetting resins such as epoxy resins are used during the conventional pull-winding method. When using this method, the resin is exposed to contaminants and foreign object damages that can cause inconsistency in resin quality.

Nowadays, manufacturers are having problems with a huge rejection rate of production. This situation is due to several issues such as those listed as follow:

- The pre-mixed resin has a finite pot life. After that, viscosity of the resin increases and the fibre impregnation process becomes progressively difficult. This means that there is a potential of the resin arrangement setting or cross-linking into a solid in the processing equipment. The cross-linked resin has to be removed prior to the resumption of production. The removal of cross-linked resin from the processing supplies can be a monotonous, time-consuming and expensive operation. Furthermore, as the ambient temperature can impact the viscosity and cross-linking rate of thermosetting resins, the low 
temperature-curable resins are not typically suitable for standard pull winding.

- The equipment needs to be methodically cleaned with a copious volume of solvent at the end of every single creation run. This creates the requirement to recoup the solvent prior to the disposal of the resin waste and to have an adequate ventilation and personal protective supplies for the workforce. The legislation also dictates the exposure limits for the workforce with regards to certain chemicals and solvents.

- Both the resin and hardener are weighed and varied manually prior to their transfer to the resin bath. During pull-winding, the resin bath has to be replenished manually. Open-top resin baths cause significant emissions of low-molecular heaviness constituents from the resin arrangement to the atmosphere.

- The excess resin remaining in the bath after the pull-winding procedure is often transferred to disposable container and allowed to cross-link to solid before disposal. Precautions have to be taken to avoid storing or cross-linking a colossal volume of varied resin in a single procedure since this can cause resin exotherming. In other words, the cross-linking reaction can come to be auto-catalytic as it proceeds. This may cause a significant rise in the temperature of the resin arrangement, leading to the thermal degradation and the emission of potentially toxic gaseous by-products.

A modified pull-winding method with close compartment processing has been designed to solve the industry issues and constraints, which is known as the automated resin infusion-fibre drying system (RIFiDS). One of the main objectives of this study is to develop a new technology that can be integrated to the current pull-winding process line. By using experimental data from a previous study, improved prototype mould has been designed and assembled on the production line. The philosophy involves the use of a resin dispensing unit where the resin and hardener are stored separately and impelled on-demand across a static mixer to a custom-designed closed resin impregnation unit. In the resin impregnation unit, the fibres are impregnated in-plane before entering the heated dye. The resin is injected alongside the fibre with extra pressure in a mould locked by a mechanical system. The constant strand glass fibre, carbon fibre or basalt fibre roving, mat, fabric or surfacing shroud is impregnated in a resin bath and then pulled (hence the term pultrusion) across a steel die by tractor mechanism. Furthermore, the latest pultrusion technology utilizes direct injection dies and the resin is introduced inside the die instead of across an external resin bath [5]. Design of the mould has been done using the CATIA V5 software package, which is a common tool to design, simulate, analyse and manufacture products in various industries including aerospace, automotive, consumer goods and also industrial machinery [6]. Common steps in designing a product include sketching, dimensioning measurement and padding into 3D [7]. CATIA V5 is able to have the better surface modelling and analysis features compared to the other modelling software [8].

Resin injection is a critical procedure as it affects the mechanical properties of the pultruded product. After the resin is injected at elevated pressure into the continuously pulled fibres, it penetrates across the fibres and simultaneously pushes the fibres away from the injection chamber wall, causing fibre compaction in the transverse direction [9]. The compaction of the fibres affects the resin penetration and hence the reinforcement complete wet out. There are two main mathematical models for resin flow in laminate. The first one is established on the theories of soil mechanics, taking the fibre bed of the laminate as a porous solid, and follows lubrication theory [10]. The models have been established on soil science that assumes a pressure gradient across vertical and horizontal direction of the laminate. Grigoriev et al. [11] have assumed the pressure gradient to be linear while few others have considered it to be nonlinear [12-13].

The resin flow in laminated composite is laminar and of low Reynolds number. The porous medium combined with a low
Reynolds number $\left(R_{\mathrm{e}} \leq 10\right)$ flow can be characterized by Darcy's Law [14]. According to Darcy's law, the resin velocity, $V$ inside the laminate is as given in Eqn. 1, where $Q$ is a volume of flow rate of resin, $K$ is permeability of the material (a measure of the ease that resin can flow through the material), $A$ is area of the cross section through which the resin is flowing, $\Delta p$ is pressure difference across a section of laminate whose length is $L, \mu$ is viscosity of the resin (dynamic) and $L$ is distance the resin has to travel.

$Q=\frac{K A \Delta p}{\mu L}$

Darcy's Law shows that the rate a resin will flow across a laminate is inversely proportional to $\mu$, viscosity of the resin. In other words, a low viscosity resin will flow faster across the dry laminate. Conversely, a high viscosity resin will flow more slowly [15] The fiber bed permeability, $K$ is analogues to the conductivity of the porous media. The more permeable is the fibre bed, the lesser confrontation resin faces to flow. According to soil science, permeability is the specific discharge each constituent hydraulic gradient. Permeability of the fibre bed may change with time due to an applied external load that changes the structures and texture of the porous bed. As the fibre volume fraction changes during compaction, therefore fibre bed permeability is a function of fibre volume fraction. Kozeny and Carman developed the semitheoretical formula for predicting the permeability of porous media as given in Eqn. 2 [16], where $\varepsilon_{\beta}$ is the porosity, $L_{C K}$ is an appropriate length scale and $C_{C K}$ is the Carman-Kozeny constant that generally takes the value of 5 .

$K_{\beta}=\frac{1}{C_{C K}} L_{C K}^{2} \varepsilon_{\beta}^{3}$

Although the length scale, $L_{C K}$ is traditionally taken as the volumetric surface area, this definition is neither unique nor necessarily the most appropriate. Some results have suggested that this Carman-Kozeny equation can be utilized to compute the permeability. In fact, experimental facts shown that this equation provides reasonable permeability estimates for random packing of spheres [17], periodic arrays of spheres and fractal porous media [18].

\section{Methodology}

Basically, the work requires the resin to be automatically metered and mixed such that a consistent batch of resin quality will be obtained. Then, the mixed resin is injected directly to the composite preforms without excessively exposing the preform to the ambient air. This will eliminate moisture absorption problem that is causing inconsistent quality of the fabricated composite part. Furthermore, the mixed resin is infused as per required only, hence it eliminates the excessive mixed resin waste. The prototype mould has been tested at actual production line at TenAsia Sdn. Bhd. Carbon fibre is provided by TenAsia Corp. Sdn. Bhd. and the stacking sequence of fibres and epoxy is also based on recommendation from TenAsia Corp. Sdn. Bhd. Both raw material identification and stacking sequence is considered as confidential by TenAsia Corp. Sdn Bhd. The close compartment has been designed initially using Perspex material as temporary mould. The mould has been designed in order to avoid preform exposure to the environment. The close compartment is also designed to reduce waste and emission for a cleaner and healthier environment. Furthermore, the prototype mould is designed to ensure resin penetration to the preform. Hence, the concept of close compartment has been chosen to avoid environmental exposure to the fabrication. The compartment is designed in a circular form to avoid resin residual and also for ease of mould maintenance by the operator. It is divided three parts for easy installation and cleaning after usage.

Dimension for the close compartment is described as follows: width is $127 \mathrm{~mm}$, length is $254 \mathrm{~mm}$ and height is $140 \mathrm{~mm}$. The TenAsia's product diameter is $38 \mathrm{~mm}$. The range of pressure sup- 
ply for the spray nozzle is 55 psi to 75 psi while the type of the nozzle is flat fan type designed with an angle of 60 degrees. This close compartment is using six nozzles, by which three nozzle at upper mould and three nozzle at the lower mould (refer Figure 2). There are two drain line with the edge of $5 \mathrm{~mm}$ to provide a better resin drain flow out. The fabric is sprayed with mixed resin and hardener at high pressure with the ratio of $1: 1$. This is to ensure that the resin inside this close compartment is not flooded with the resin spray because both bottom and upper part of the mould have nozzles. The wastage resin will flow to the collective bag.

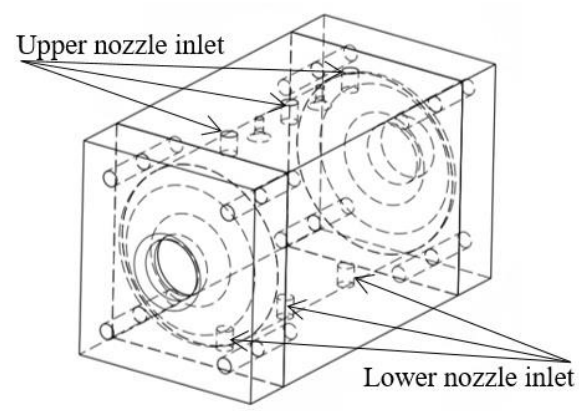

Fig. 1: Design of the close compartment mould

Fiberglass carbon preform is used during the trial run in the actual production line (refer Figure 3). The mould has been installed by sections starting with the front section, middle section and followed by end section into the line production. Then, all sections are combined together by locking mechanism whereby both front and end section are assembled to the middle section. In order to prevent the mould from moving along with the mandrel, the front section of the mould has been locked by a stopper that is fitted to the line. After the mould has been installed properly, the nozzles are connected to hose and the end of the hose is connected to the pressure chamber. The mixed resin/hardener is poured into the pressure chamber. Before the process is started, the pressure chamber needs to be secured by locking it to prevent any leakage of the resin. This trial has been conducted while the pulling mechanism is switched on and the machine is pulling the preform to forward movement. While the preform is moving, the resin are sprayed out by the nozzle inside the mould to the preform. The trial is using 2, 4 and 6 nozzles to determine the most suitable numbers of nozzles to be used to ensure full of the preform. For each nozzle, the pressure selected for testing is at 10, 20 and 40 psi, respectively. The spray pattern and the level of resin penetration to the preform surface core are recorded for every 30 seconds for a total duration of 120 seconds.

\section{Results and discussion}

Figure 4 shows the two sets of paired nozzles on the close compartment processing, which are 2 and 4 nozzles. For 2 nozzles, the area covered is $140.904 \mathrm{~cm}^{2}$ at $20 \mathrm{psi}$ for 30 seconds duration. On the other hand, for 4 nozzles, the area covered increases to 144.02 $\mathrm{cm}^{2}$ at 20 psi for 30 seconds duration. The difference in the area covered between 4 nozzles and 2 nozzles is about $2.2 \%$. The difference is not too much but it will affect the end product result. For 2 nozzles, the resin does not adequately penetrate to the product core and only covered the surface of product. Meanwhile for 4 nozzles, the penetration of resin has been good and it is fully covered the product to its core.

Figure 5 shows three sets of paired nozzles on the close compartment processing, which are 2, 4 and 6 nozzles. For set of 2 nozzles, it indicates that the area covered is $154.432 \mathrm{~cm}^{2}$ at 30 seconds duration with 40 psi pressure. In contrast, 4 nozzles indicates the area covered is $168.91 \mathrm{~cm}^{2}$ at 40 psi for 30 seconds duration. For the set of 6 nozzles, the area covered is $110.998 \mathrm{~cm}^{2}$ at $40 \mathrm{psi}$ for 30 seconds duration. As the result, a set of 4 nozzles is the best set of pair because at 40 psi for 30 seconds duration, it can cover more $9.4 \%$ than 2 nozzles and more $52.2 \%$ than 6 nozzles. Based on the observation, the set of 2 nozzles, the resin only covers at the bottom of the product while for 6 nozzles, the pressure turbulence builds up too rapidly inside the closed compartment mould that can disturb the process by turning the resin into mist. A set of 4 nozzles is the best because it provides a good covering of resin to the product.

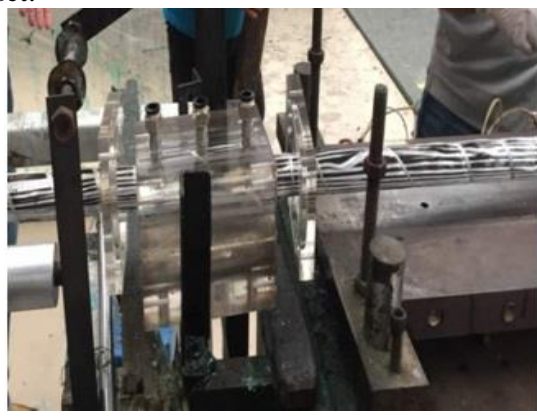

(a)

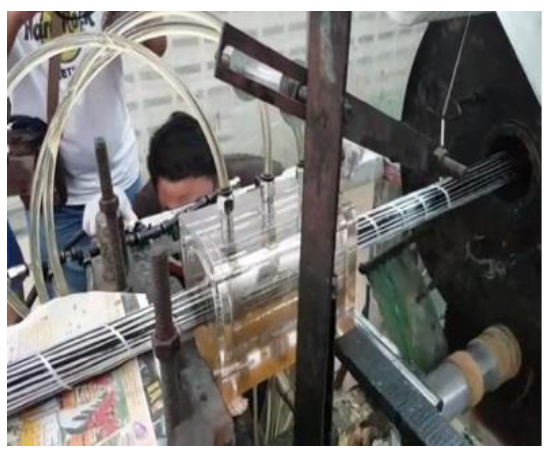

(b)

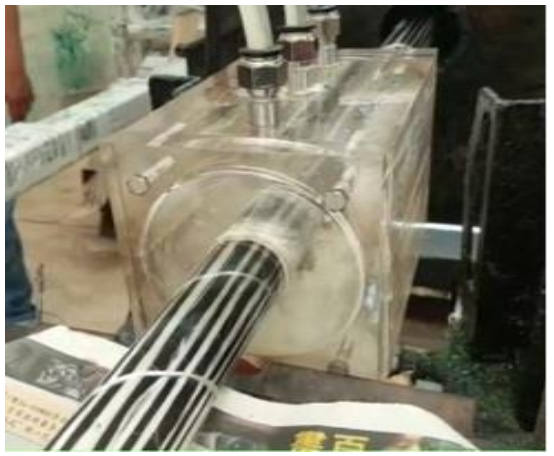

(c)

Fig. 2: Processes at the actual line manufacturing; (a) Installation of mould (b) Connecting resin hose (c) Resin introduced inside the mould Spray comparison ( 30 s)

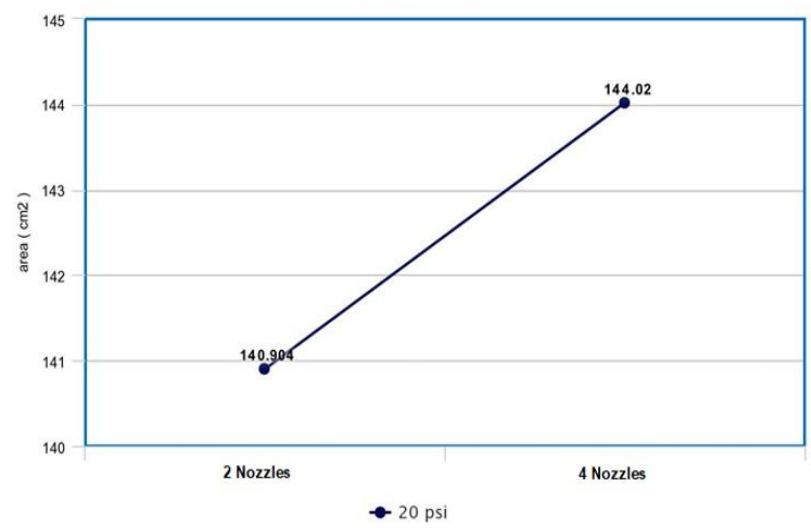

Fig. 3: Result for preform area coverage at 20 psi using 2 and 4 nozzles at 30 seconds 


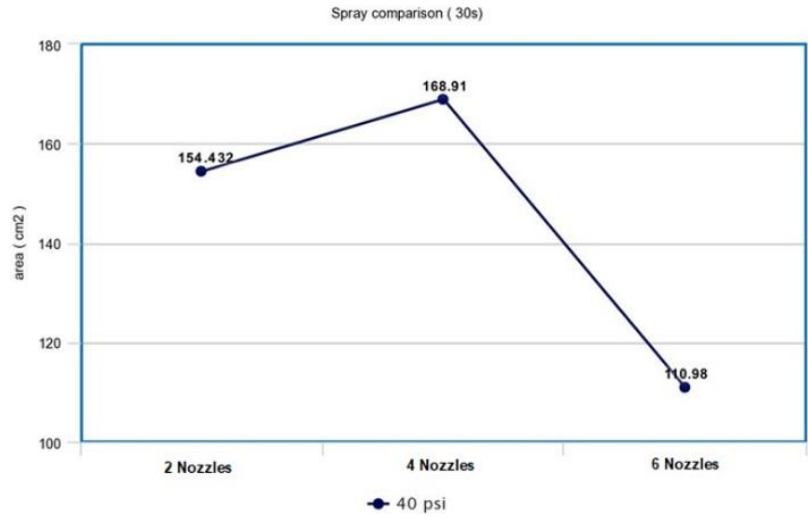

Fig. 4: Result for preform area coverage at 40 psi using 2, 4 and 6 nozzles at 60 seconds

Figure 6 shows the two sets of paired nozzles on the close compartment processing, which are 2 and 4 nozzles. For a set of 2 nozzles, it indicates the area covered is $256.72 \mathrm{~cm}^{2}$ at $20 \mathrm{psi}$ for 60 seconds duration while for a set of 4 nozzles, the area covered is $294.5 \mathrm{~cm}^{2}$ at $20 \mathrm{psi}$ for 60 seconds duration. It shows that the set of 4 nozzles is more efficient by covering more than $14.7 \%$ compared to the set of 2 nozzles. Based on the observation, the set of 2 nozzles cannot provide the resin to cover up and penetrate into the product until to the core. On contrary, for the set of 4 nozzles, it provides good covering and the resin penetration until to the core. An increase in numbers of nozzle has resulted in an increase in volume of resin entering the mould compartment, hence resulted in an increase in preform permeability and resin areas of coverage. The limitation will be the resin pot life, whereby the whole system cures by time.

Spray comparison (60s)

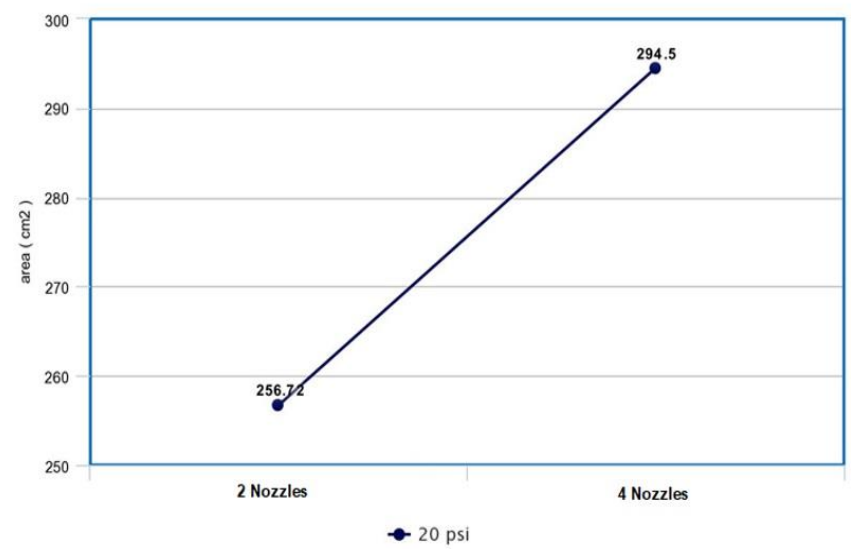

Fig. 5: Result for preform area coverage at 20 psi using 2 and 4 nozzles at 60 seconds

Observations have been made on the preform after the processes are conducted. There are various conditions on the preform because of the reaction on the different numbers of nozzles. The method that brings a good result is the 4 numbers of nozzles that is used in the processes. The pressure that is used on 4 nozzles is suitable to ensure the resin capsulate the preform. The resin is fully capsulate the preform as shown as Figure 7. Another observation has been made on the result of the other preform that reacts on 2 nozzles that spray the resin. The end result of the preform shows the resin did not fully capsulate because of the lack of pressure and the number of nozzles is not enough to ensure the resin capsulate and fully covers the preform until the core. Figure 8 shows the preform does not received enough resin to capsulate the preform until the core. By adding the number of nozzles into the process, the result on the preform might be changed. It is because of the different pressure in the same time and the different total of resin that the preform received between the nozzles. Process using 6 nozzles gives a high total resin to the preform but the mist inside the close compartment is high too. The high formation of mist can disturb the process of resin to penetrate the preform until the core as shows in Figure 9. It may result the rich resin on the preform surface, which is not good to the quality of the end product [19].

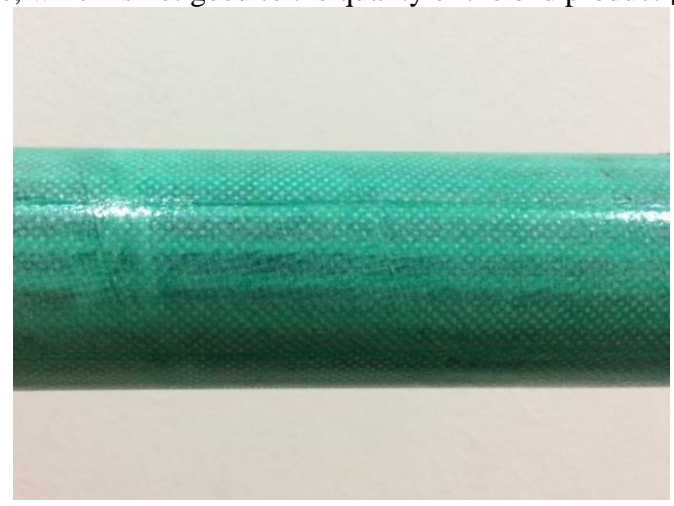

Fig. 6: Resin fully capsulate the preform

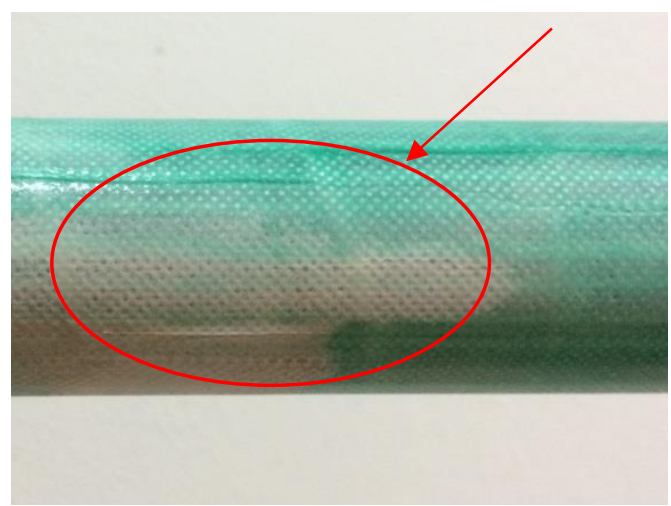

Fig. 7: Resin does not penetrate until the core of the preform and resulted in poor resin

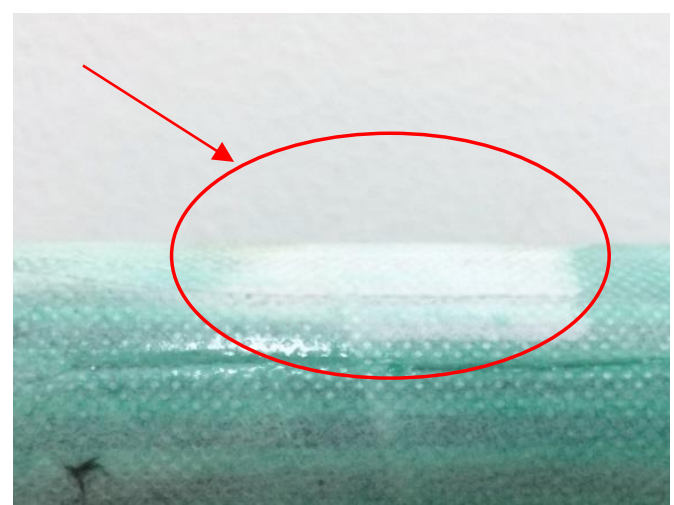

Fig. 8: Formation of mist inside close compartment disturbs the resin to penetrate

\section{Conclusion}

This stage of investigation involves finding and comparing between experimental data to the actual condition of the manufacturing pull-winding system. This investigation has involved the integration of close compartment, e.g. Perspex mould, with fully operational pull-winding production line. In conclusion, the most economical and productive design in this preliminary study of automated resin infusion-fiber drying system (RIFiDS) is identified to be a spray type with 2 upper and lower nozzle run on appropriate pressure of $20 \mathrm{psi}$. This setting works better due to deeper resin penetration in shorter time due to less mist and very low dead area inside the closed compartment. The design of the close compartment mould is to avoid the exposure of the preform to the environment. 


\section{References}

[1] AVK Task Force (2010), Sustainability of Fibre-Reinforced Plastics: An Assessment Based on Selected Examples of Application. Federation of Reinforced Plastics

[2] Bechtold G \& Lin Y (2003), Influence of fiber distribution on the transverse flow permeability in fiber bundles. Composites Science and Technology 63(14), 2069-2079

[3] Reynolds N \& Pharaoh MW (2009). An introduction to composites recycling in Management, Recycling and Reuse of Waste Composites. Oxford: Woodhead Publishing Ltd.

[4] Witten E \& Schuster A (2010), Composites Market Report: Market Developments, Challenges and Chances. Federation of Reinforced Plastics

[5] Correia J (2013), Pultrusion of advanced fiber-reinforced polymer (FRP) composites. Woodhead Publishing Series in Civil and Structural Engineering, 207-251

[6] Louis R, Virgilio Q, Robert P, Frederick V \& Fawzi K (2010), Will model-based definition replace engineering drawings throughout the product lifecycle? A global perspective from aerospace industry Computers in Industry 61(5), 497-508

[7] Michal F, Puskar M, Boslai R, Kopas M, Stefan K \& Robert H (2018), Design of experimental vehicle specified for competition Shell Eco-marathon 2017 according to principles of car body digitisation based on views in 2D using the intuitive tool Imagine \& Shape CATIA V5. Advances in Engineering Software 115, 413-428

[8] Pawar SS, Patil NV \& Shete HV (2017), Stress analysis of leaf spring by using photo elasticity technique. International Research Journal of Engineering and Technology 4(12), 1475-1478

[9] Mei P, Hao W, Joong H, Chun K, Kin T, Leng J \& David H (2012), Critical factors on manufacturing processes of natural fiber composites. Composites Part B: Engineering 43(8), 3549-3562

[10] Velthem PV, Ballout W, Daoust D, Sclavons M, Cordenier F, Henry E, Dumont D, Destoop V, Pardoen T \& Bailly C (2015), Influence of thermoplastic diffusion on morphology gradient and on delamination toughness of RTM-manufactured composites. Composites Part A: Applied Science and Manufacturing 72, 175183

[11] Grigoriev S, Krasnovskii A \& Kazakov I (2014), The impact of pre-heating on pressure behavior in tapered cylindrical die in pultrusion of large-sized composite rods. Advanced Materials Research 1064, 120-127

[12] Sanchez J, Guerrero J, Varas D \& Puenta J (2016), Experimental analysis of ice sphere impacts on unidirectional carbon/epoxy laminates. International Journal of Impact Engineering 96, 1-10

[13] Ghnatios C, Chinesta F \& Binetruy C (2015), 3D modeling of squeeze flows occurring in composite laminates. International Journal of Material Forming 8(1), 73-83

[14] Kundu P, Kumar V \& Mishra I (2016), Experimental and numerical investigation of fluid flow hydrodynamics in porous media: Characterization of pre-Darcy, Darcy and non-Darcy flow regimes. Powder Technology 303, 278-291

[15] Centea T \& Hubert P (2012), Modelling the effect of material properties and process parameters on tow impregnation in out-ofautoclave prepregs. Composites Part A: Applied Science and Manufacturing 43(9), 1505-1513

[16] Valdes-Parada F, Ochoa-Tapia J \& Ramirez J (2009), Validity of the permeability Carman-Kozeny equation: A volume averaging approach. Physica A: Statistical Mechanics and its Applications 388(6), 789-798

[17] Bryant S, King P \& Mellor D (1992), Network model evaluation of permeability and spatial correlation in a real random sphere packing. Transport in Porous Media 11(1), 53-70

[18] Lemaitre R \& Adler P (1990), Fractal porous media IV: Threedimensional stokes flow through random media and regular fractals. Transport in Porous Media 5(4), 325-340

[19] Hirano Y, Yamane T \& Todoroki A (2016), Through-thickness electric conductivity of toughened carbon-fiber-reinforced polymer laminates with resin-rich layers. Composites Science and Technology 122, 67-72 